University of Nebraska - Lincoln

DigitalCommons@University of Nebraska - Lincoln

Faculty Publications from the Department of Electrical \& Computer Engineering, Department Electrical and Computer Engineering

$11-1-2001$

\title{
Mechanical, geometrical, and electrical characterization of silicon membranes for open stencil masks
}

\author{
E. Sossna \\ Institute of Technological Physics, IMA, University of Kassel, Heinrich-Plett-Strasse 40, 34132-Kassel, \\ Germany
}

A. Degen

University of Kassel

I. W. Rangelow

University of Kassel

M. Drzik

Slovak Academy of Sciences, Dubravska 9, 84220 Bratislava, Slovak Republic

P. Hudek

Slovak Academy of Sciences, Dubravska 9, 84220 Bratislava, Slovak Republic

See next page for additional authors

Follow this and additional works at: https://digitalcommons.unl.edu/electricalengineeringfacpub

Part of the Electrical and Computer Engineering Commons

Sossna, E.; Degen, A.; Rangelow, I. W.; Drzik, M.; Hudek, P.; Tiwald, T. E.; and Woollam, John A., "Mechanical, geometrical, and electrical characterization of silicon membranes for open stencil masks" (2001). Faculty Publications from the Department of Electrical and Computer Engineering. 26.

https://digitalcommons.unl.edu/electricalengineeringfacpub/26

This Article is brought to you for free and open access by the Electrical \& Computer Engineering, Department of at DigitalCommons@University of Nebraska - Lincoln. It has been accepted for inclusion in Faculty Publications from the Department of Electrical and Computer Engineering by an authorized administrator of DigitalCommons@University of Nebraska - Lincoln. 


\section{Authors}

E. Sossna, A. Degen, I. W. Rangelow, M. Drzik, P. Hudek, T. E. Tiwald, and John A. Woollam 


\title{
Mechanical, geometrical, and electrical characterization of silicon membranes for open stencil masks
}

\author{
E. Sossna, ${ }^{\text {a) }}$ A. Degen, and I. W. Rangelow \\ Institute of Technological Physics, IMA, University of Kassel, Heinrich-Plett-Strasse 40, 34132-Kassel, \\ Germany \\ M. Drzik and P. Hudek \\ Slovak Academy of Sciences, Dubravska 9, 84220 Bratislava, Slovak Republic
}

T. E. Tiwald and J. A. Woollam

J. A. Woollam Company, Incorporated, 645 M Street, Suite 102, Lincoln, Nebraska 68508

(Received 1 June 2001; accepted 17 September 2001)

\begin{abstract}
Silicon membranes are used for stencil masks which are key to charged particle projection lithography, particularly for ion projection lithography, electron beam projection. Quantitative and qualitative determination of the mechanical properties of the true thickness, thickness variations (morphology), electrical conductivity and stress is critical to the development of next generation lithography. The metrology setup includes high accuracy thickness, refractive index and electrical conductivity measurement based on infrared variable angle spectroscopic ellipsometry, thickness variation characterization based on the Fizeau interferometric scheme and mechanical stress evaluation based on a novel double bulging technique. (C) 2001 American Vacuum Society.
\end{abstract}

[DOI: $10.1116 / 1.1417548$ ]

\section{INTRODUCTION}

For determination of the true thickness with sufficient accuracy and precision $(5 \mathrm{~nm})$, we demonstrate a technique based on infrared variable angle spectroscopic ellipsometry (IR-VASE®). ${ }^{1}$ The spectral range employed, from 2 to 33 $\mu \mathrm{m}$, and varying angles of incidence $\left(60^{\circ}, 65^{\circ}\right.$, and $\left.70^{\circ}\right)$, allow the IR-VASE method to be very sensitive to the thickness of layers as well as to the concentration and profile of $\mathrm{Si}$ membrane doping. The refractive index of the Si membrane was evaluated using a Drude model for the free-carrier effects $^{2}$ and is based on consideration of a superimposed Sellmeier model. ${ }^{3}$ Due to the high value of the refraction index of silicon in the visible region, a favorable fringe (Fizeau interferometric scheme) value is obtained. Using a digital image processing evaluation of the intensity variations by interpolating the interference fringe data, a high resolving power in the nanometer range can be obtained. By combining such a relative thickness data evaluation with ellipsometric (IR-VASE) point-wise absolute thickness measurements, very sensitive precise mapping of the membrane thickness variation can be made. The stress was measured with the double bulging technique (deflecting the membrane in both directions), ${ }^{4}$ which is based on the well known bulging method. It allowed us to increase the measurement accuracy $(<0.1 \mathrm{MPa})$ of the method and to investigate low stress membranes $(<2 \mathrm{MPa})$.

\section{SILICON MEMBRANE FABRICATION USING THE $P N$-JUNCTION ELECTROCHEMICAL ETCH STOP TECHNIQUE}

Thin $(<10 \mu \mathrm{m})$ membranes are mostly fabricated with a wet chemical process in which silicon is etched by aeqeous

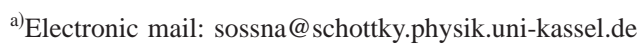

alkali solutions tetramethyl ammonium hydroxide (TMAH) ${ }^{5}$ The etch mechanisms ${ }^{6}$ are based on the oxidation of silicon in water, and the neutral silicon hydroxide diffuses from the solid surface, building silicon hydroxide complexes in the ambient with excessive $p \mathrm{H}$ values.

$$
\mathrm{Si}+4 \mathrm{OH}^{-} \rightarrow \mathrm{Si}(\mathrm{OH})_{4}+4 e^{-} \rightarrow \mathrm{SiO}_{2}(\mathrm{OH})_{2}^{2-}+2 \mathrm{H}^{+}+4 e^{-} .
$$

The excess electrons in the conducting band of the silicon crystal reduce water molecules on the surface, producing hydrogen gas and hydroxide ions which react with the hydrogen ions afterwards to form water (as a cycle).

$$
\begin{aligned}
& 4 \mathrm{H}_{2} \mathrm{O}+4 e^{-} \rightarrow 4 \mathrm{OH}^{-}+2 \mathrm{H}_{2} \uparrow, \\
& 2 \mathrm{H}^{+}+2 \mathrm{OH}^{-} \rightarrow 2 \mathrm{H}_{2} \mathrm{O} .
\end{aligned}
$$

The different etch rates of silicon in alkali solution depend on the crystal orientation if everything else (e.g., temperature or concentration) is constant. The electrochemical etch stop (ECES) technique seems to be a process that is easily executed for the fabrication of membranes with moderate residual stress. ECES is based on anodic passivation of silicon in an aqueous alkali solution. A positive potential of $0.5-0.8$ $\mathrm{V}$ over the open circuit potential can passivate both $p$ - and $n$-type silicon from etching. A sufficient anodic potential causes accumulation of holes $\left(\mathrm{h}^{+}\right)$at the silicon surface, which increases the oxidation states of silicon atoms at the silicon-electrolyte interface. ${ }^{5}$ The $\mathrm{Si}^{2+}$ combines with $\mathrm{OH}^{-}$ to form hydroxide. Subsequently $\mathrm{Si}(\mathrm{OH})_{2}$ forms silicon dioxide by releasing hydrogen.

$$
\mathrm{Si}+2 \mathrm{~h}^{+}+2 \mathrm{OH}^{-} \rightarrow \mathrm{Si}(\mathrm{OH})_{2} \rightarrow \mathrm{SiO}_{2}+\mathrm{H}_{2}
$$

Therefore, the surface is covered with thin but dense $\mathrm{SiO}_{2}$ which, reduces the etch rate of silicon by about two orders of magnitude. Also, the etching stops when the front side mem- 


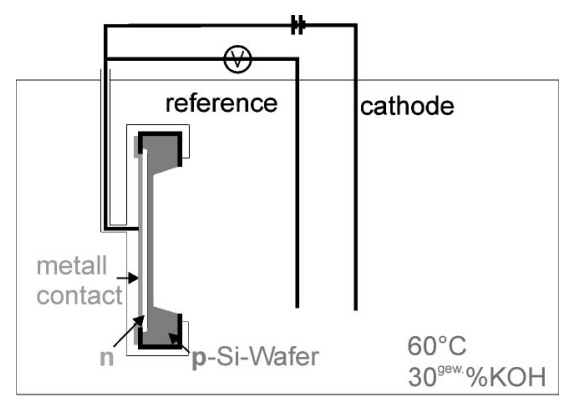

FIG. 1. Experimental setup with a reverse bias voltage on the $p n$ junction for the electrochemical etch stop technique.

brane approaches the space-charge region, because more $\mathrm{OH}^{-}$will be accelerated toward the surface of the membrane and buildup silicon dioxide. By using a reverse biased $p n$ junction structure (Fig. 1), the thin $n$-type $\mathrm{Si}$ region can be anodically passivated from the etch process, but the $p$-Si substrate (due to a potential drop at the $p n$ junction) is insufficiently protected and is etched with TMAH. The thickness of the membrane and how much it varies depends here on the dopant (type and concentration) and on the applied bias voltage, ${ }^{1}$ which influences the space-charge region, since it works through the $p n$ wafer flow process. It follows that the thickness of the membrane as well as the stress depends only on the doping concentration $N_{a}$ for the $p$ side and $N_{d}$ for the $n$ side. However the membrane thickness can vary due to the barrier potential $\phi_{B}$. With the depth of the $n$-type dopant (phosphorus or arsenic), $d_{n}$, known and from Eq. (1) we get the relationship for the effective membrane thickness: ${ }^{1}$

$$
d=d_{n}+\sqrt{\frac{2 \epsilon_{s}}{q N_{a}} \frac{N_{d}}{N_{d}+N_{a}}\left(\phi_{B}+U\right) .}
$$

Consequently, we can compensate with doping concentrations $N_{a}$ for the $p$ side and $N_{d}$ for the $n$ side not only for the stress, but also for the thickness of the membrane, while changing it due to the bias voltage $U$. In this way wellcontrolled repeatable thin silicon membranes were produced.

\section{THICKNESS MEASUREMENT USING IR-VASE}

The samples were measured using a commercially available infrared variable angle spectroscopic ellipsometer (Fig. $2) .^{7}$ The instrument consists of a Fourier transform-based infrared spectrometer combined with a rotating-compensator variable angle ellipsometer. The compensator not only ensures the accuracy of the ellipsometric angle $\Delta$ over its full $360^{\circ}$ range, but it can also measure the amount of depolarization caused by the sample. For this study, data were acquired over the 2-30 $\mu \mathrm{m}\left(330-5000 \mathrm{~cm}^{-1}\right)$ spectral range. The beam diameter is $8 \mathrm{~mm}$ at the sample, with a $3^{\circ}$ angular spread. Data were taken with a resolution of $32 \mathrm{~cm}^{-1}$ at incidence angles of $60^{\circ}, 65^{\circ}$, and $70^{\circ}$. This yielded ellipsometric data with reasonable sensitivity and signal-to-noise ratios. Values for $\Psi$ and $\Delta$ at each wavelength comprise the ellipsometric spectrum, and are sensitive to the various layers and to the microstructure of the sample. ${ }^{8,9}$ In general,

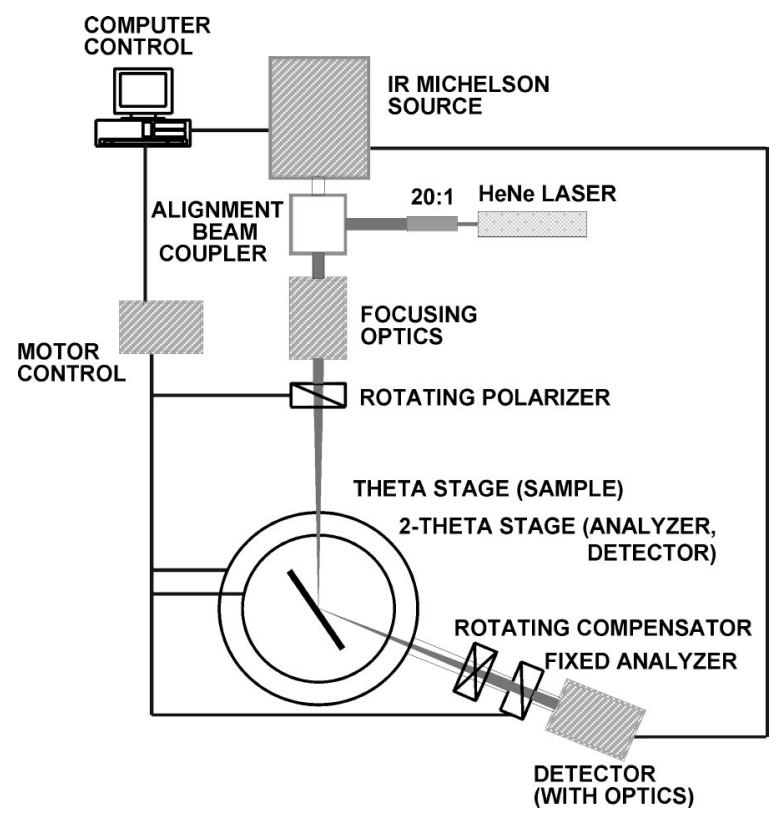

FIG. 2. IR-VASE® setup.

ellipsometric spectra cannot be inverted; therefore, information must be extracted by optimizing an appropriate optical model to fit the data. The nonlinear Levenberg-Marquardt numerical regression procedure was used (described by Herzinger et al., in Ref. 3) it adjusted the various parameters until the mean (weighted) squared error between the calculated and measured ellipsometric values was minimized. One output of the fitting program is the standard $90 \%$ confidence limit, which is calculated during the regression procedure. The objective accuracy of the $90 \%$ confidence limit depends on (among other things) the minimum of systematic error in both the data and in the optical model. ${ }^{9}$ The optical properties of each silicon layer are defined by the classical Drude equation $^{10}$ combined with a Sellmeier model:

$$
\begin{aligned}
& \epsilon_{j}=\text { offset }+\frac{A}{E_{a}^{2}-E^{2}} \frac{1}{\rho_{\mathrm{dc}_{j}}} \frac{4 \pi \hbar^{2}}{\left(E^{2} \tau+i \hbar E\right)}, \\
& \rho_{\mathrm{dc}_{j}}=\frac{m^{*}}{N_{j} e^{2} \tau}=\frac{1}{N_{j} e \mu},
\end{aligned}
$$

where $\epsilon_{j}$ is the complex dielectric dispersion of the $j$ th layer. The offset and $A$ and $E_{a}$ terms comprise a Sellmeier model for the residual dielectric response from the interband transitions. The $E$ term is the energy of the incident photons, and $\tau$ is the mean scattering time of the free carriers. The quantity $\rho_{\mathrm{dc} j}$ is the dc resistivity of the $j$ th layer. It is inversely proportional to the electronic charge $e$, the carrier concentration $N_{j}$, and the carrier mobility $\mu\left(\mu=e \tau / m^{*}\right.$ where $m^{*}$ is the carrier's effective mass). For this study, we assume that $\tau$ is independent of the photon energy. The resistivity profile of a sample can be directly determined from Eq. (2). The free carrier profile $N_{j}$ can also be determined if one accounts for the dependence of resistivity on both $N_{j}$ and the mobility $\mu$, since $1 / \rho=e \mu N_{j}$. This can be difficult because $\mu$ $=e \tau / m^{*}$, and both $m^{*}$ and $\tau$ are strong functions of doping 
a) Depth profile of dielectic constants $\varepsilon_{1}$ and $\varepsilon_{2}$ at $500 \mathrm{~cm}^{-1}$ :

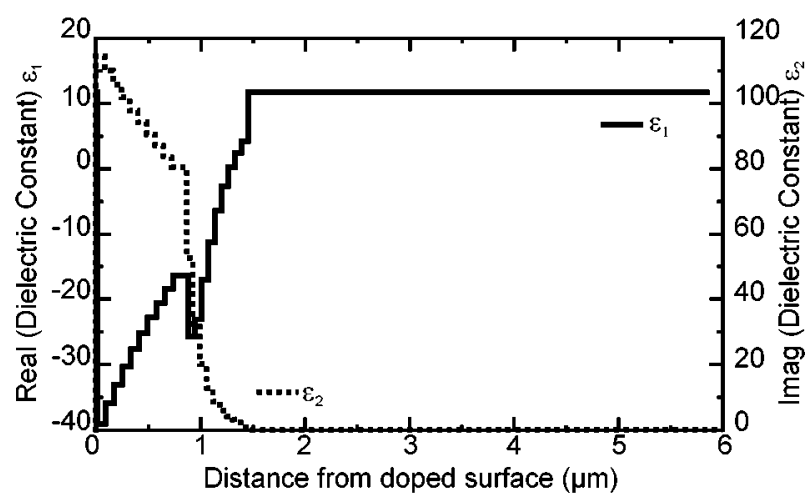

b) Resulting free carrier depth profile:

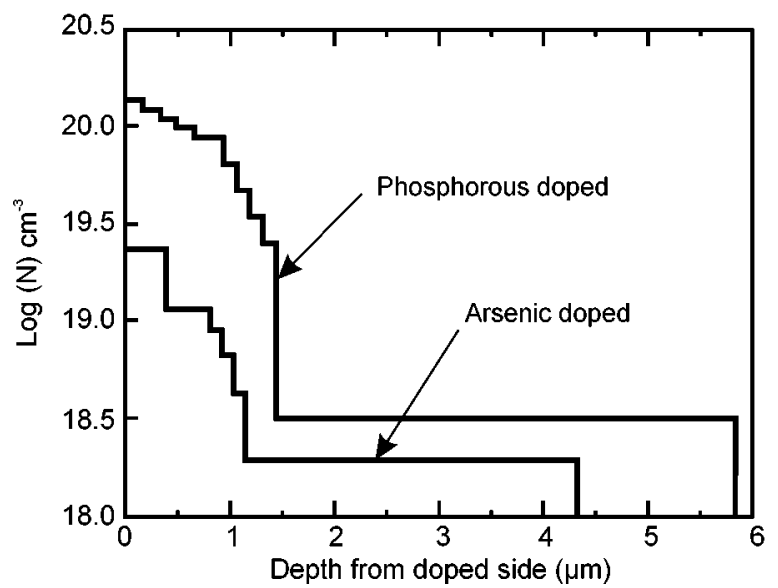

FIG. 3. (a) Depth profiles of dielectric constants $e 1$ and $e 2$ at $500 \mathrm{~cm}^{-1}$ for the phosphorus doped $p n$ membrane. The profiles were determined from a model of IR-VASE data. (b) Resulting free carrier concentration dependent on the membrane depth from the front (doped) side.

in heavily doped silicon [Fig. 3(b)]. Here, our primarily interest is the total film thickness; therefore, we only need to apply the resistivity model described by Eq. (3). Like in a previous study, ${ }^{1}$ IR-VASE measurements were made on both the front (etched side) and back of the membrane, because the highly doped region strongly absorbs infrared light. Simultaneous analysis of both data sets produces the graded optical dielectric response model shown in Fig. 3(a) for a phosphorus doped $p n$ membrane. Note that Fig. 3(a) only shows the dielectric response to a single wavelength (500 $\mathrm{cm}^{-1}$ ). For any given wavelength, the shape of this profile is defined by the dielectric response function of Eq. (2). Models of the two samples are shown in Table I using thicknesses determined from the data fit. The $90 \%$ confidence interval (see the discussion above) for the combined thickness of the doped and undoped portions of the silicon membrane was $\pm 0.04 \mu \mathrm{m}$ or better. As measured by infrared spectroscopic ellipsometry, the total sample thicknesses for the phosphorus doped and the arsenic doped layer were 5.88 \pm 0.04 and $4.53 \pm 0.02 \mu \mathrm{m}$, respectively (Table I).

\section{THICKNESS VARIATION}

In order to measure thickness variations of thin transparent silicon membranes, the method of shadow/phase visual-
TABLE I. Layer thicknesses of the M002-4-Es-5 V and M988-141Es-As membranes, determinated by IR-VASE®. [The error bars represent a $90 \%$ confidence interval of the data, assuming no systematic errors (Ref. 3).]

\begin{tabular}{lcc}
\hline \hline & Phosphorus doped $(\mu \mathrm{m})$ & Arsenic doped $(\mu \mathrm{m})$ \\
\hline Native oxide etched side & $0.01 \pm 0.001$ & $0.001 \pm 0.001$ \\
Silicon bulk region & $4.432 \pm 0.008$ & $3.30 \pm 0.01$ \\
Silicon with free carriers & $1.44 \pm 0.03$ & $1.26 \pm 0.01$ \\
Native oxide "back" & $0.018 \pm 0.003$ & $0.008 \pm 0.001$ \\
Total thickness & $5.88 \pm 0.04$ & $4.53 \pm 0.02$ \\
\hline \hline
\end{tabular}

ization is employed. In the case of $p n$ membranes the light passing through the membrane is scattered by microscopic surface roughness, creating a coherent light speckled pattern. As a result, the contrast in the interference pattern is nearly zero, and the interference technique cannot be used to observe the surface topology. In the experiments membranes with thicknesses varying from 4 to $6 \mu \mathrm{m}$ were tested, with one specular and one diffuse surface. The root mean square of this opposite side roughness was $R_{q} \cong 0.03 \mu \mathrm{m}$. Thus, we have been forced to examine principles of optical filtration and also phase and shadow visualization procedures. Besides the high measuring sensitivity, a characteristic feature of shadow or phase visualization techniques is the simplicity of their scheme realization. The common optical scheme of the experiments is drawn in Fig. 4(a). In the setup, a laser diode $650 \mathrm{~nm} / 10 \mathrm{~mW}$ serves as a point-like source, and the beam of plan-parallel rays is collimated by a well corrected lens. Passing through the transparent membrane the rays deviate in accordance with thickness changes. Classic optical filtration can be achieved by putting a binary filter a circular diaphragm, into the focal plane of the filtering lens so that the higher spatial frequencies are stopped. Thus, the speckled structure in the image is depressed. In the experiments, doublet lenses with focal length $f=265 \mathrm{~mm}$ were used as were filter diaphragms $0.1-0.5 \mathrm{~mm}$ in diameter. In addition to filtering of the speckled structure, waviness irregularities of the membrane profile can also be visualized by appropriately choosing the diameter of a circular hole. Another frequently used shadow method is the widely used testing method of optics aberrations, the Foucault knife. This method is based on a sharp edge filter placed into the light beam after it has passed through the phase object. ${ }^{11}$ By shading part of the light in the focal plane, the image of the object becomes overlapped by a shadow pattern. The method is very sensitive and, in a good aperture, where aberration free lenses are used, surface deviations of the order of $\lambda / 100$ can be identified. The good sensitivity of the measurement is demonstrated also in Fig. 4(b), where deviations of the membrane planarity of tens of nanometers are visible. The optical scheme was arranged the same way as in previous tests of samples with diffuse-like surfaces. There were no filters used in the Fourier transform plane, but the objective lens of a charge coupled device (CCD) camera was able to be shifted out of the plane of the sharp image. By defocusing the imaging system, the phase changes of the transparent object are easily observable. This phenomenon arises from the fact that 
a)

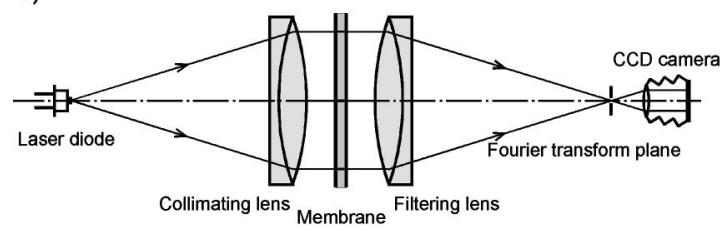

b)

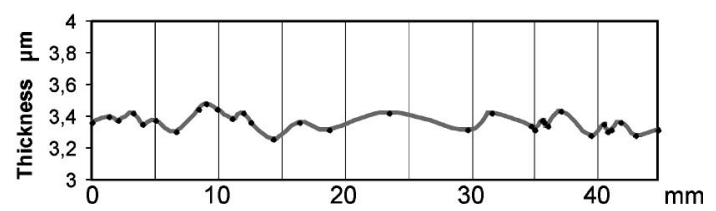

c)

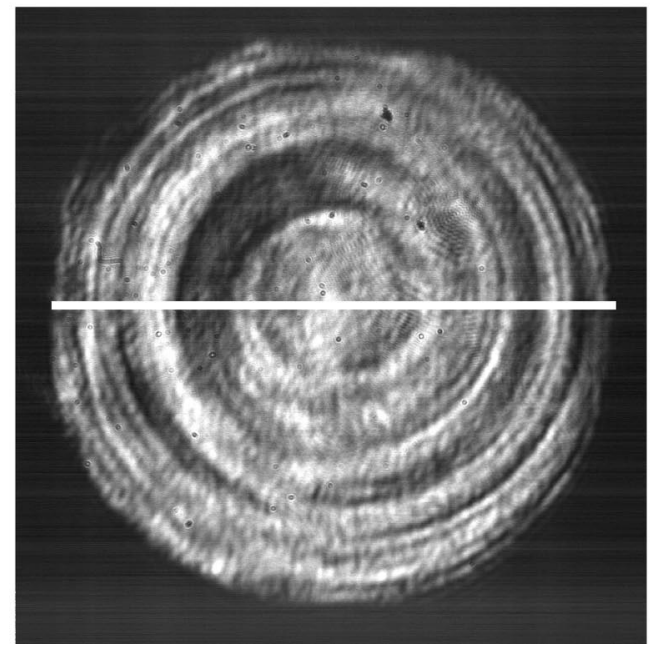

FIG. 4. (a) Scheme of optical filtering and shadow/phase visualization. (b) Thickness cross section of the membrane. (c) CCD picture of the Foucault shadow pattern of the membrane measured. The thickness profile was taken on the line.

the inverse Fourier transform did not take place completely at the position shifted by the value of $b$ from the position of the image plane. The intensity distribution at the plane of visualization can be described as ${ }^{12}$

$$
I(x, y)=1+\frac{\lambda}{2 \pi} b\left(\frac{\partial^{2} \varphi(x, y)}{\partial x^{2}}+\frac{\partial^{2} \varphi(x, y)}{\partial y^{2}}\right),
$$

where $\lambda$ is the wavelength of light and $\varphi(x, y)$ is the object phase function. From this equation it follows that the second derivatives of the membrane thickness variation can be visualized and, hence, the radii of curvature of the object surfaces can also be observed. On account of that, by mapping the intensity distribution throughout the membrane area, the thickness variations can be even quantitatively evaluated with a CCD camera [Fig. 4(c)]. Estimation of the resolving power leads to values of $20-40 \mathrm{~nm}$ in membrane thickness variation, which can be reliably determined.

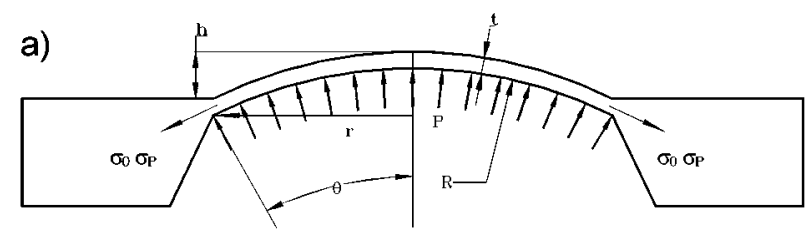

b)

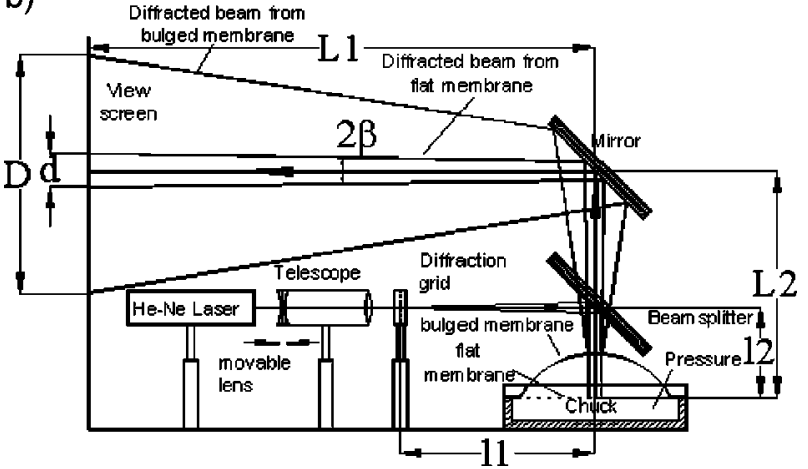

FIG. 5. (a) Bulging method: $p$ : pressure difference; $t$ : film thickness; $r$ : membrane radius; $\sigma_{0}$ : residual stress; $\sigma_{p}$ : induced stress; $R$ curvature radius of the sphere; $h$ deflection of the membrane in the center. (b) Setup of the diffraction image method; $d$ zero level or the flat membrane distance and $D$ bulged membrane distance between the diffraction patterns.

\section{STRESS DETERMINATION WITH THE DOUBLE BULGING METHOD}

The bulging method is widely used for determination of the mechanical characteristics of silicon membranes. With this method the silicon membrane with radius $r$, thickness $t$, and initial stress $\sigma_{0}$ is clamped directly at the wafer ring close to the membrane, and pressure $p$ is applied to one side, shown in Fig. 5(a). The membrane forms a segment of a sphere with radius $R$; thus the deflection of the membrane center from its initial position is $h$. The pressure-deflection curve obtained using this method represents functions of mechanical properties, initial stress, and membrane geometry. ${ }^{13-15}$ The governing equation is given as

$$
p \cong \frac{4 t \sigma_{0}}{r^{2}} h+\frac{8 t}{3 r^{4}} Y k h^{3},
$$

where $k$ is a correction factor, $k=1-0.24 v-0.00027$ ( 1 $-v) \sigma$. By fitting the measured membrane deflection values $h$ for the applied load pressure $p$ to bulging Eq. (5), it is possible to determine the average residual stress $\sigma_{0}$ of the membrane and the value of biaxial modulus $Y$. A setup for the diffraction imaging method, which was used for displacement detection, is shown schematically in Fig. 5(b). It consists of a light source (a He-Ne laser), a telescope, a diffraction grid, a membrane holder (chuck) and a projection system that includes a beamsplitter, a mirror and a viewing screen. The divergent laser beam is collimated in the telescope to focus sharply on the viewing screen. From the laser the beam is diffracted by the diffraction grid and forms a spatial diffraction pattern, which is projected onto the membrane by the beamsplitter. The reflected image is projected onto the viewing screen by a mirror. If the membrane bulges 
a)
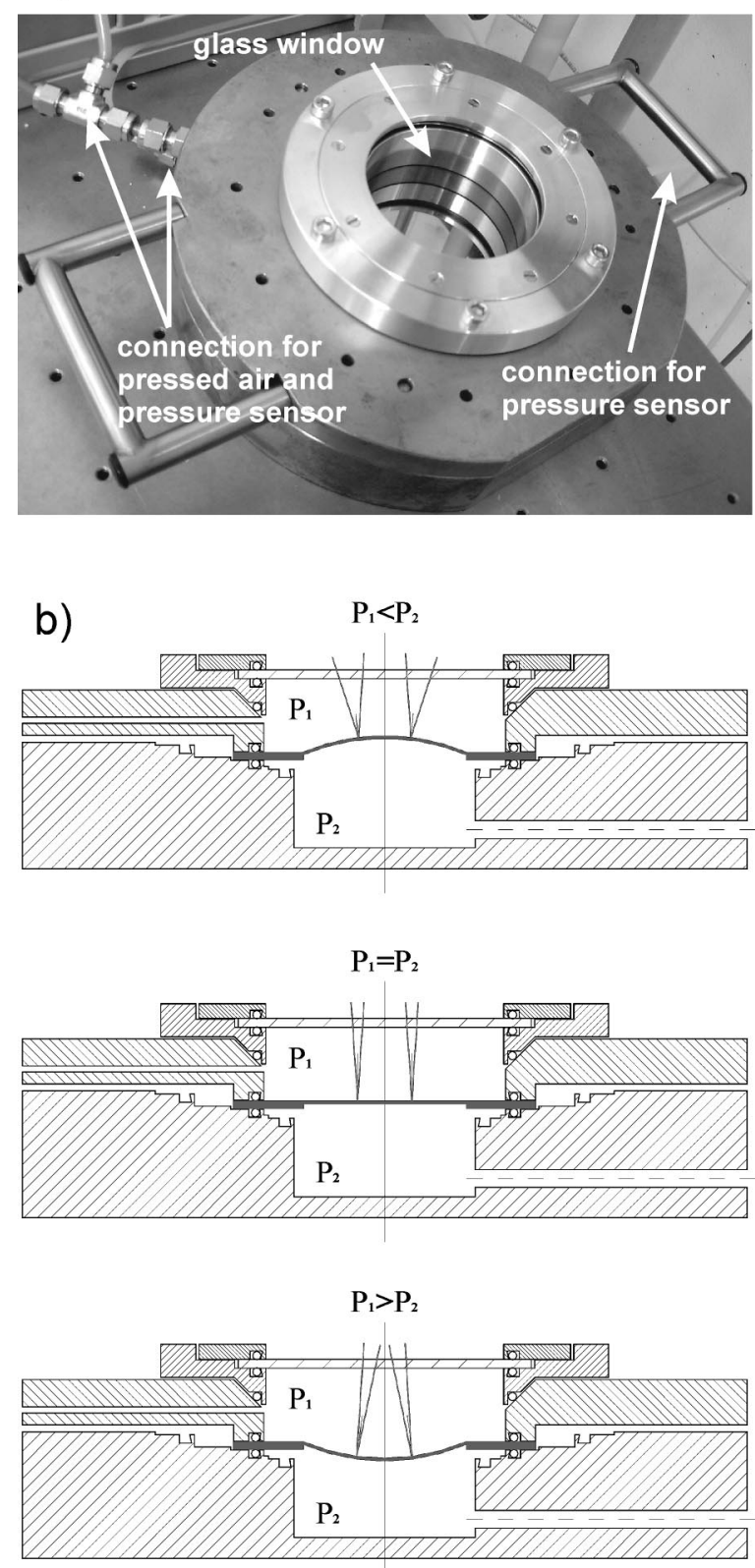

FIG. 6. (a) Membrane holder (chuck) for the double bulging method; (b) membrane positions in the chuck and the paths of incident and reflected laser beams for overpressure zero-position (where overpressure is compensated for by gravitational force) and underpressure states.

then the distance between the diffraction, patterns will increase from the initial $d$, zero level or the flat membrane, to the $D$, bulging membrane. The membrane displacement $h$ can be then expressed as

$$
h=\frac{r^{2}(D-d)}{8 L l \beta},
$$

where $l=l 1+l 2, L=L 1+L 2, r$ is the radius of the membrane and $\beta$ is the diffraction angle. The advantage of this method is that the zero level $d=2(L+l) \tan \beta$ depends only on the geometry of the setup, the diffraction grid constant and the wavelength of the laser but not on the membrane
Tensile stress of the arsenic doped pn-membrane:

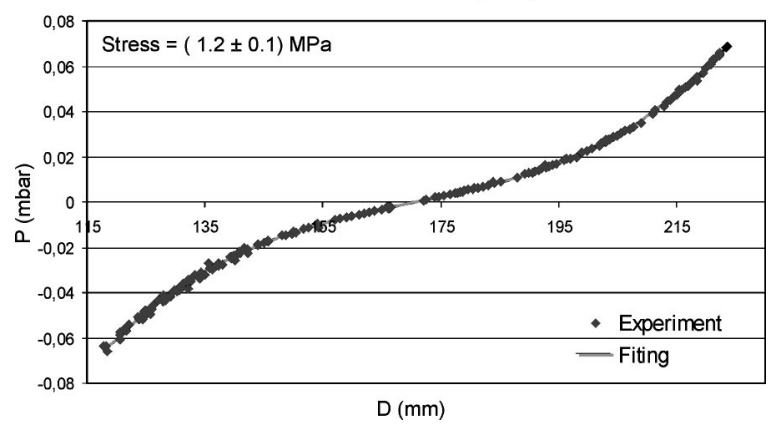

FIG. 7. Experimental data from the double bulging experiment on the 4.53 $\mu \mathrm{m}$ thick $50 \mathrm{~mm}$ diam arsenic doped $p n$ membrane.

itself, the so-called "geometrical" zero-level method. In the double bulging technique the membrane is deflected in both directions, ${ }^{16}$ applying over- or underpressure in a specially designed double chamber [Fig. 6(a)]. Figure 6(b) shows the positions of the membrane in the chuck and the path of the incident and reflected laser beams for overpressure, zero position (where overpressure is compensated for by gravitational force) and underpressure states. The data collected in the experiment will be fitted by means of an improved least square fit. ${ }^{4}$ The use of this method allowed us to get better resolution from the fitting and less scattering of the measured values, the latter due to outer local air pressure variations. Applied in the region of higher pressure, where the elastic term contributes greatly to Eq. (5), the double bulging method makes it possible to determine the fitting of the zero level from the antisymmetry of the pressure-deflection curve (Fig. 7). The comparison of fitted, geometrical and measured with reference plane plate zero levels, provided in previous experiments, ${ }^{4}$ has shown the perfect coincidence of all these values and it opens the possibility to use such a comparison for fine tuning diffraction image optics. The stress measured in the $4.53 \mu \mathrm{m}$ thick $p n$ membrane was $1.2 \mathrm{MPa}$ and was determined within an accuracy of $0.1 \mathrm{MPa}$.

\section{SUMMARY}

Quantitative and qualitative determination of the mechanical properties of the true thickness, thickness variation (morphology), and electrical conductivity was demonstrated. An interferometric method of precise thickness variation measurement of silicon membranes was presented. With regard to point-wise thickness measurements, which were carried out by the ellipsometric method, the procedure offers a very sensitive tool with which to map thickness variations. Moreover, using the principles of shadow, as well as phase contrast visualization, the possibility of mapping the thickness variations was also proven on membrane samples with surfaces that diffusely scatter light. By evaluating the intensity distribution throughout the area of the membrane image using defocusing, an attempt was made to determine the absolute values of membrane thickness variations. Moreover, the application of the double bulge technique for nonabsolute displacement detection techniques, such as interferometry, 
offers a method for zero-level determination and it can eliminate systematical errors connected with those techniques in the subsequent calculations of elastic constants.

\section{ACKNOWLEDGMENTS}

This research work was performed within the framework of Project No. 01MBE 22/97, Future Lithography, supported by the German government (Bundesministerium für Bildung und Forschung). Additional support was provided by the U.S. National Science Foundation under SBIR Contract No. 9901510 .

${ }^{1}$ E. Sossna, R. Kassing, I. W. Rangelow, C. M. Herzinger, T. E. Tiwald, J. A. Woollam, and Th. Wagner, J. Vac. Sci. Technol. B 18, 3259 (2000).

${ }^{2}$ T. E. Tiwald, D. W. Thompson, J. A. Woollam, W. Paulson, and R. Hance, Thin Solid Films 313-314, 661 (1998).

${ }^{3}$ C. M. Herzinger, B. Johs, W. A. McGrahan, J. A. Woollam, and W. Paulson, J. Appl. Phys. 83, 323 (1998).

${ }^{4}$ A. Degen, N. Abedinov, T. Gotszalk, E. Sossna, M. Kratzenberg, and I. W. Rangelow, Proc. MEE 57-58, 425 (2001).
${ }^{5}$ I. W. Rangelow, Deep Etching of Silicon (Politechnica, Warsaw, Poland, 1996), p. 8.

${ }^{6} \mathrm{H}$. Seidel, L. Csepregi, A. Heuberger, and H. Baumgaertel, J. Electrochem. Soc. 137, 3612 (1990).

${ }^{7}$ IR-VASE® system, J. A. Woollam Co., Inc., Lincoln, NE.

${ }^{8}$ R. M. A. Azzam and N. M. Bashara, Ellipsometry and Polarized Light (North-Holland, New York, 1977), Chap. 4, p. 274.

${ }^{9}$ C. M. Herzinger, P. G. Snyder, B. Johs, and J. A. Woollam, J. Appl. Phys. 77, 1715 (1995).

${ }^{10}$ C. R. Pidgeon, in Handbook on Semiconductors, edited by T. S. Moss and M. Balkanski (North-Holland, Amsterdam, 1980), Vol. 2, pp. 227-230.

${ }^{11}$ D. Malacara, Optical Shop Testing (Wiley, New York, 1978).

${ }^{12}$ L. M. Soroko, Hilbert Optics (Nauka, Moscow, 1981) (in Russian).

${ }^{13}$ A. Degen, F. Shi, E. Sossna, R. Sunyk, J. Voigt, B. Volland, B. Reinker, and I. W. Rangelow, Proc. SPIE 3665, 113 (1999)

${ }^{14}$ A. Degen, J. Voigt, E. Sossna, F. Shi, I. W. Rangelow, E. Haugeneder, and H. Löschner, Proc. SPIE 3996, 97 (2000).

${ }^{15}$ A. Degen, B. Volland, E. Sossna, F. Shi, M. Kratzenberg, and I. W. Rangelow, University of Kassel Internal Report (1999).

${ }^{16}$ A. Degen, J. Voigt, M. Kratzenberg, F. Shi, J. Butschke, H. Löschner, R. Kaesmaier, A. Ehrmann, and I. W. Rangelow, Proc. SPIE 3997, 395 (2000). 